\title{
Exploring the impact of providing men with information about potential prostate cancer treatment options prior to receiving their biopsy results
}

\section{Brindha Pillay, ${ }^{1,2,3}$ Daniel Moon, ${ }^{4,5,6}$ Jeremy Grummet, ${ }^{5,7,8}$ Denny Meyer, Helen Crowe ${ }^{1,2,10}$ Sarah Mann, ${ }^{5}$ Nicholas Howard, ${ }^{1}$ Addie Wootten, ${ }^{1,10}$ Mark Frydenberg ${ }^{5,8,11,12}$}

1. Epworth Prostate Centre, Epworth Healthcare, Victoria, Australia 2. Urology, Royal Melbourne Hospital, Victoria, Australia

3. Peter MacCallum Cancer Centre, Victoria, Australia

5. Australian Urology Associates, Victoria, Australia

7. Urology, Alfred Health, Victoria, Australia

\section{Introduction}

$>$ Prostate cancer $(\mathrm{PCa})$ is one of the most commonly diagnosed cancers in men worldwide $>$ Receiving a diagnosis of localised PCa requires men to make decisions regarding treatment choices based on preferences and medical advice [2]

$>71-76 \%$ of patients consider only one treatment option and may make a treatment choice almost immediately after a cancer diagnosis ${ }^{[3,4]}$ due to anxiety about cancer progression

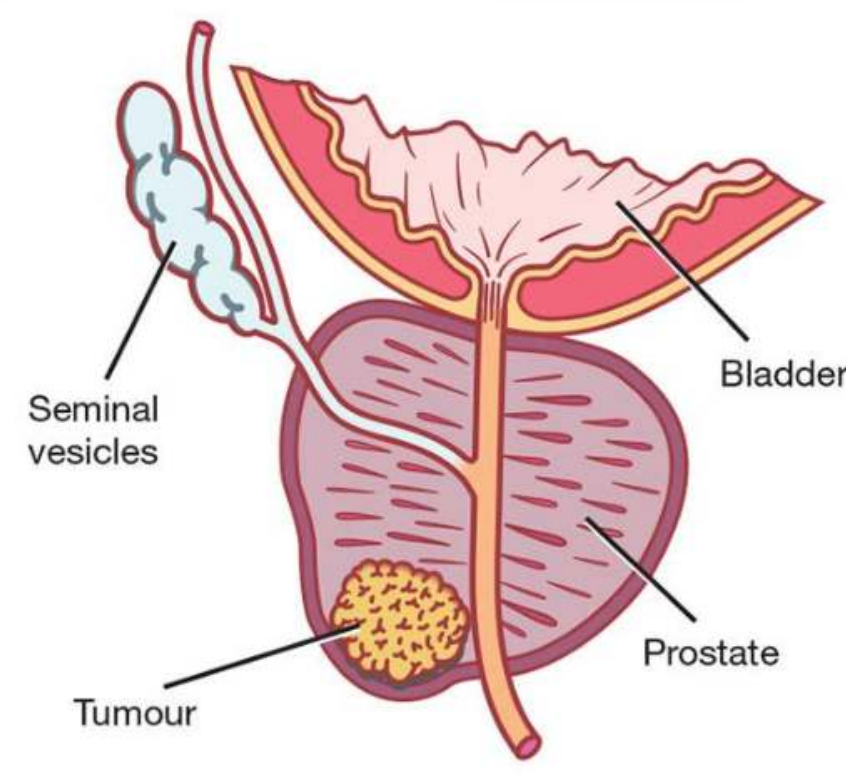

Figure 1. Localised prostate cancer, where cancer cells have

Higher anxiety levels pre-biopsy have been associated with lesser knowledge of $\mathrm{PCa}$ treatment options [5]

$>$ Few studies have explored the impact of providing men with information about $\mathrm{PCa}$ treatment options before biopsy [6].

\section{Aim}

To determine:

$>$ Whether Australian patients found it acceptable to receive information about PCa treatment options at time of biopsy referral, before receiving a diagnosis

$>$ Whether receiving this information at this time influenced changes in anxiety, depression and distress from pre- to post-biopsy, and impacted treatment decisional conflict post-diagnosis

\section{Methods}

\section{Inclusion criteria}

1. Men referred for an initial prostate biopsy for an elevated PSA or suspicious digital rectal exam

2. Men not suspected of having metastatic cancer

> Between June 2016 and September 2017, 126 consecutive patients who met eligibility criteria were invited to participate in the study.

$>98$ returned the baseline questionnaire (response rate $=78 \%$ )

\section{Procedure}

> All patients received a standard biopsy instruction sheet. A randomised block design was used to allocate patients into the intervention or control group.

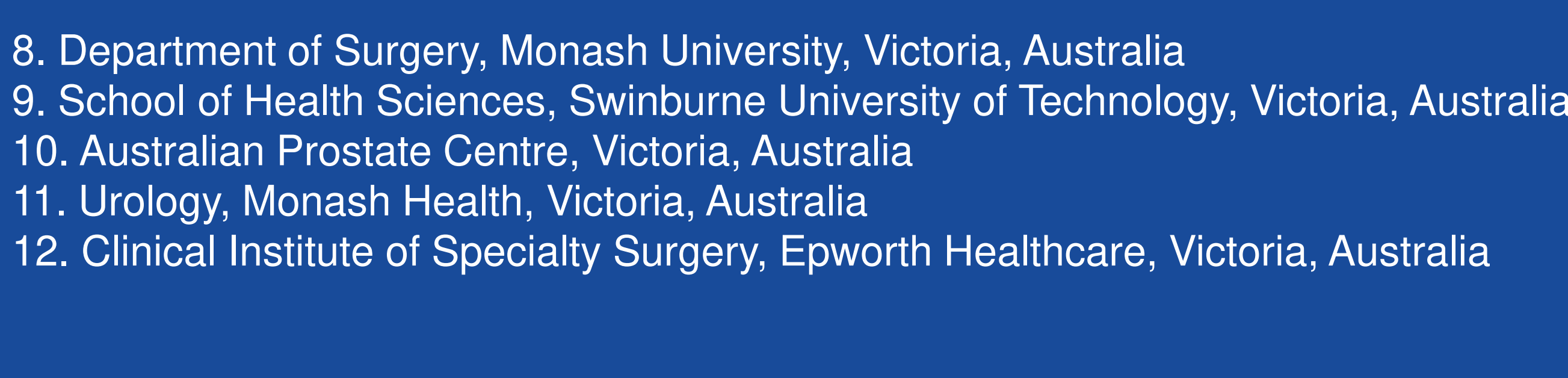

$>$ Patients in the intervention group were provided with an information booklet about treatment options.

> Questionnaires were completed pre-biopsy (Time 1) and at 2-3 weeks post-biopsy (Time 2).

\section{Measures}

$>$ Distress Thermometer (DT)

> Generalised Anxiety Disorder-7 (GAD-7)

> Patient Health Questionnaire (PHQ-9),

$>$ Decisional Conflict Scale

Acceptability of the intervention, and usefulness of the booklet in improving patient understanding was assessed at Time 2

\section{Intervention}

$>$ Intervention booklet is second in a series of 4 developed by the Prostate Cancer Foundation Australia for men with localised PCa

$>$ Written as a standalone booklet

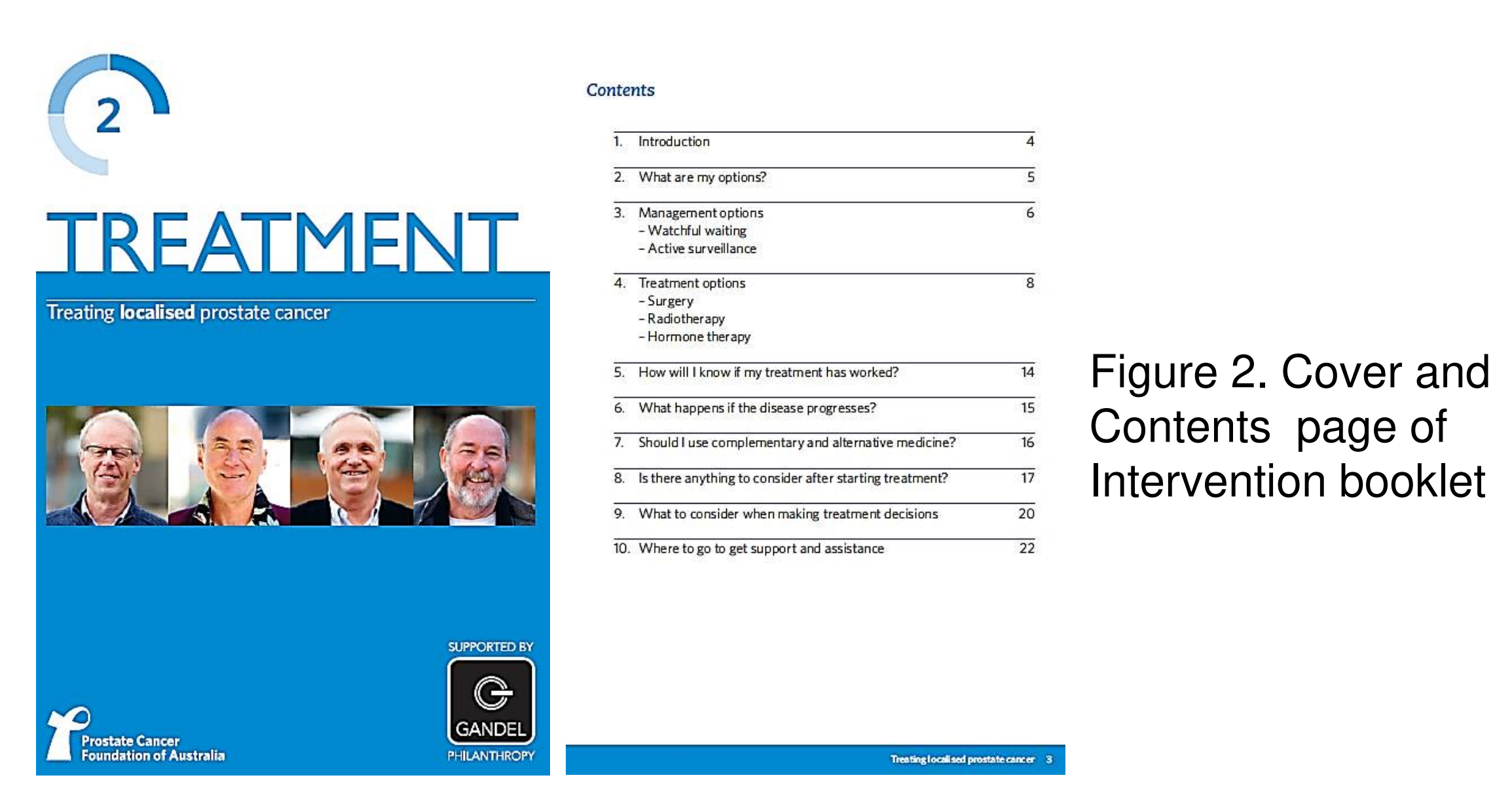

\section{Statistical Analyses}

$>$ Controlling for baseline differences and attrition propensity, changes from baseline to follow-up in anxiety, depression, and distress scores were compared for the two groups using an intention-to-treat mixed model repeated measures (MMRM) analysis assuming $\mathrm{AR}(1)$ dependence.

> Appropriate transformations were applied to the DT, $P H Q-9$, and GAD-7

$>$ Paired t-tests were used to assess if there were significant changes in scores in the intervention and control groups

\section{Results}

$>N=98$, Mean $(\mathrm{SD})$ age $=64.6(7.1)$ years

$>$ No significant differences between the control ( $n=40)$ and intervention $(n=58)$ groups across demographic characteristics and baseline scores.

> $82 \%$ of men were married, $92 \%$ were Caucasian, $67 \%$ received a positive biopsy result

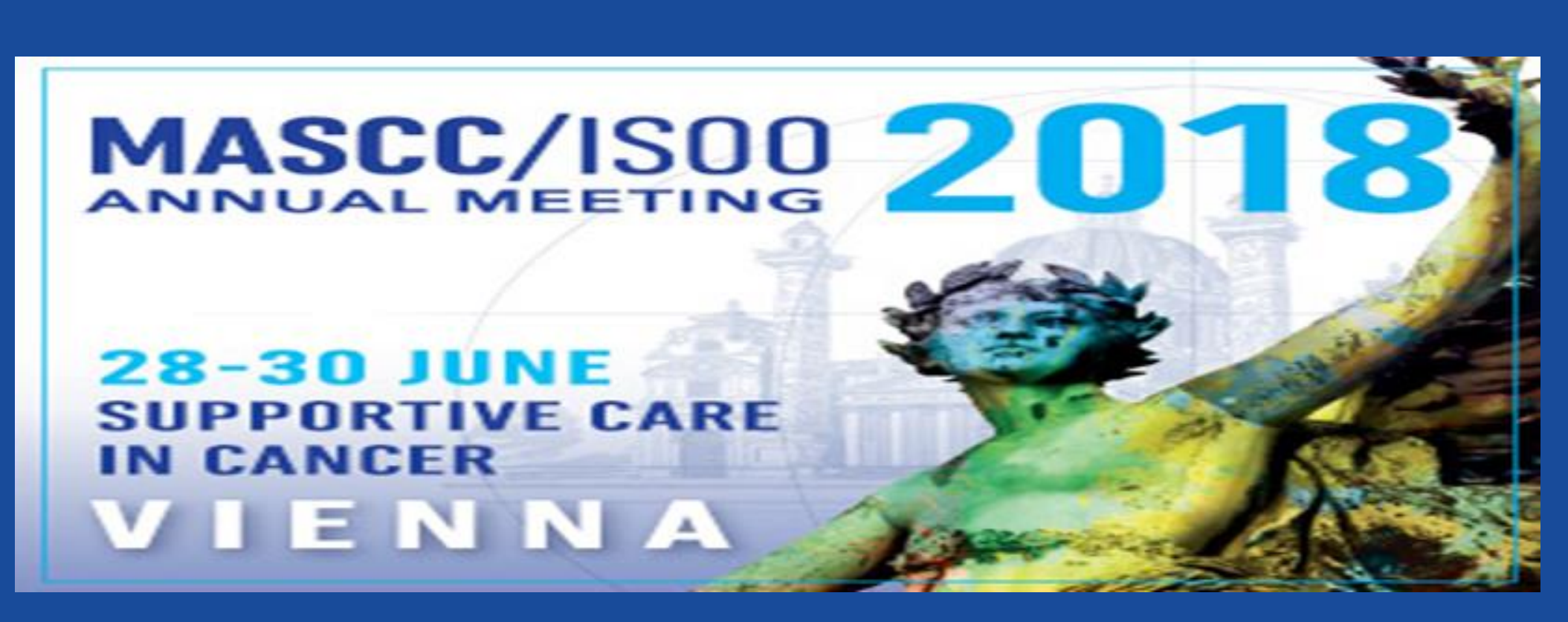

MMRM analyses showed no significant differences between control and intervention groups in changes in symptoms ( $p>.05)$ from pre- to post-biopsy. Table 2: Time 1 and Time 2 Scores and Change Score Analyses (Time 2
minus Time 1) for Control and Intervention Groups

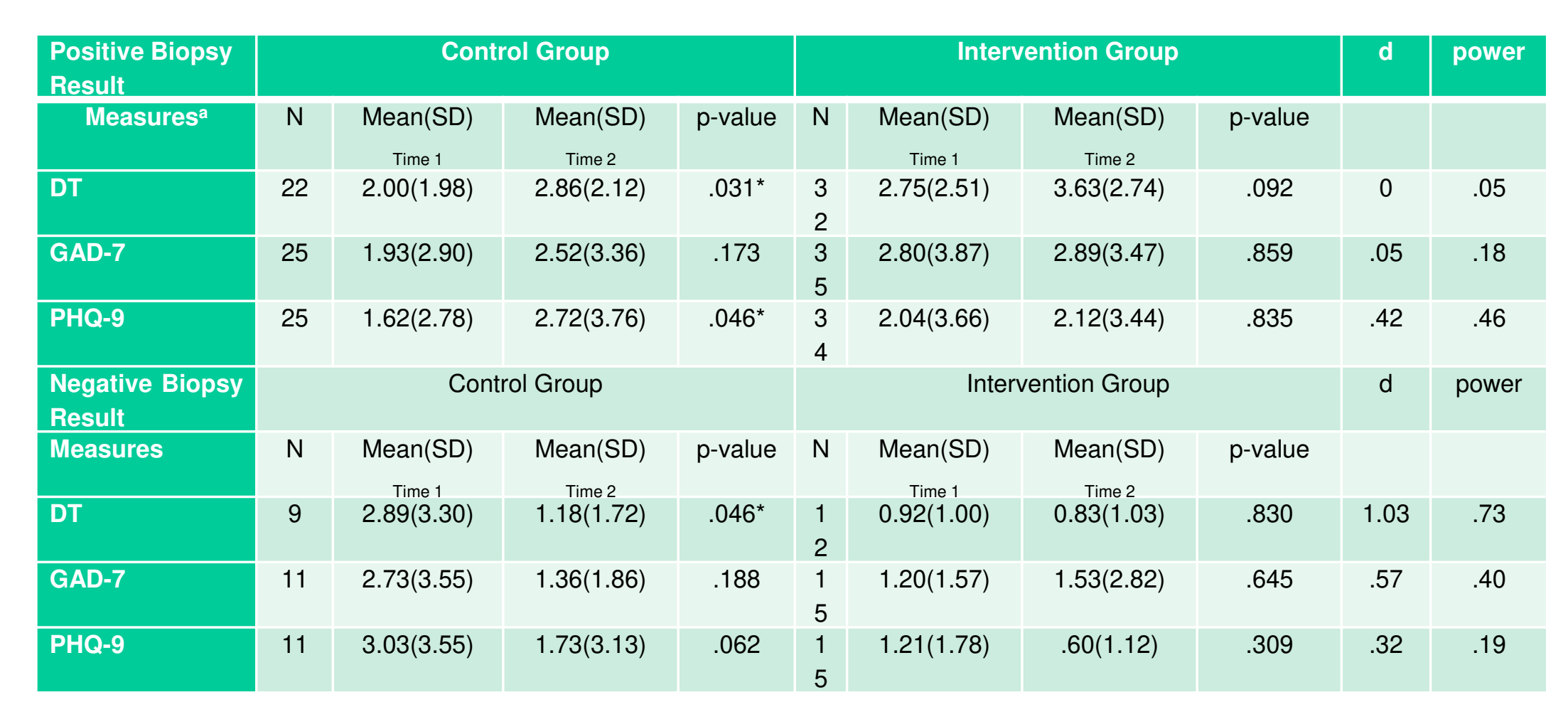

\section{Control group:}

$>$ Patients with a positive result had significant increases in distress and depressive symptoms from pre- to post-biopsy.

$>$ Patients with a negative result had a significant decrease in distress and trend towards decrease in depressive symptoms.

Intervention group:

$>$ No significant changes from pre- to postbiopsy on any of the measures.

$>$ Treatment decisional conflict did not differ significantly between the groups

\section{Acceptability and usefulness of intervention}

$98 \%$ of patients who read the booklet stated they preferred to be given such information

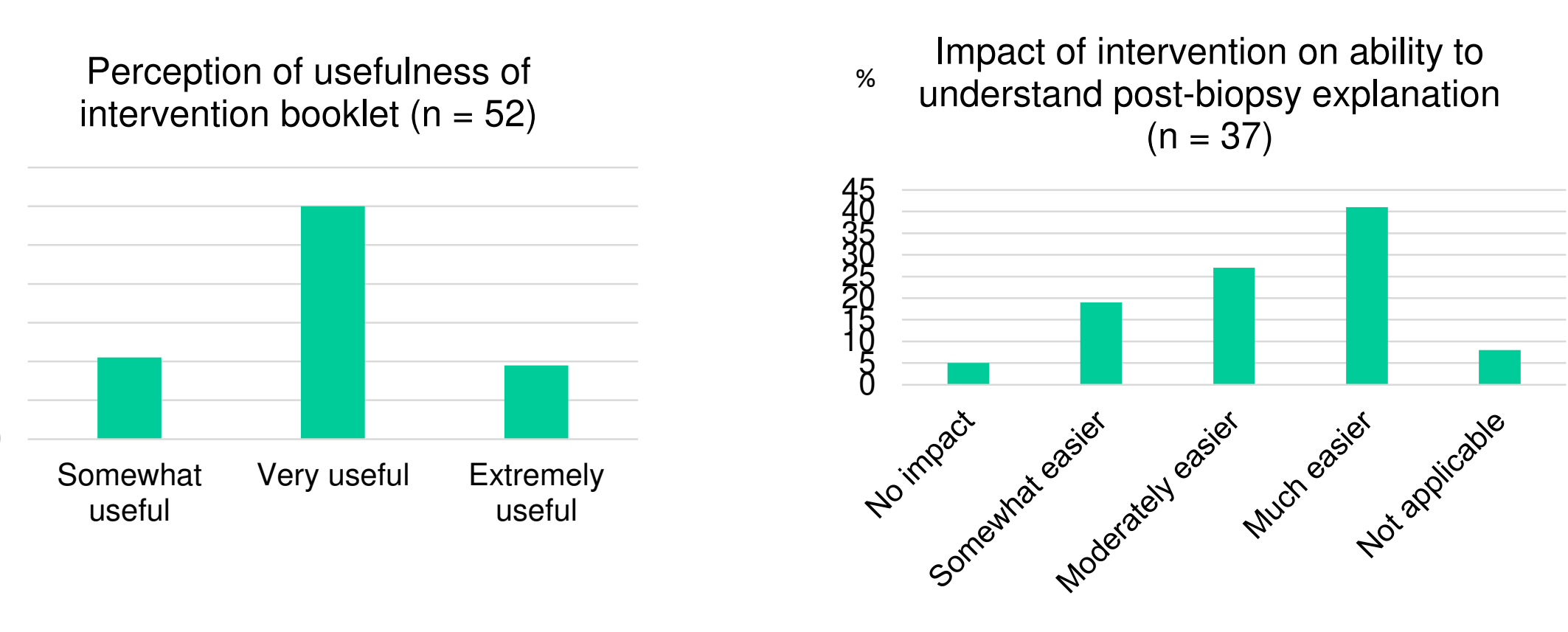

\section{Conclusions}

Providing information about PCa treatment options prior to diagnosis did not impact on anxiety, depression, distress or decisional conflict

$>$ Changes in psychological symptoms may be influenced by various factors including physician-patient communication and management of patient expectations

> Limitations: Small number of patients in the positive biopsy group; possibly underpowered to detect changes in symptoms across time

Patients preferred to be given information about treatment options pre-biopsy as it eliminated the need to search for information from other sources 